\title{
Herd Behaviour as an Incentive Scheme*
}

\author{
Nicolas Melissas \\ Appendix one with Marc Pauly \\ $\mathrm{IAE}(\mathrm{UAB})$ and ECARES(ULB)
}

May 1999

\begin{abstract}
This paper presents a model of technology invention in an emerging market. Managers wait and adopt the standard technology in the hope to free-ride on the effort level of another manager who may invent a superior technology. The more managers who adopt the standard technology, the more their successors believe that probably the superior technology doesn't exist. As this hampers the successors' incentives to innovate, herding in my model reduces the scope of strategic waiting.

JEL classification: D83,D82,D62

Keywords: Information externality, herd behaviour, free-rider problem, social learning

\footnotetext{
${ }^{*}$ Correspondence can be sent to Nicolas Melissas (melissas@cc.uab.es). I am very grateful to my thesis advisor M.Dewatripont for his many helpful suggestions. This paper also greatly benefited from comments made by P.Bolton, P.Legros and two anonymous referees. I thank seminar participants at ECARE, DELTA, IAE and GREMAQ. I also benefited from comments made by A.Banerjee, M.Castanheira, J.Gyntelberg, M.Ruckes and J.Zwiebel. Financial support from the Interuniversity Poles of Attraction Programme, Contract PAI P4/28 is gratefully acknowledged. Finally I am also very grateful to M.Castanheira for his many encouragements at the start of my research work.
} 


\section{Introduction}

People often infer information out of the actions of other people. For example, when making their purchasing decisions consumers often choose the most popular brand because they think that its popularity indicates a better price/quality combination $^{1}$. People do not go and eat in an empty restaurant, because they believe that the food quality is low. When arguing with someone, try to strengthen your argumentation by claiming that everybody agrees with you on that point. This trick (even if it is not true) often succeeds in convincing more reluctant minds that you are right.

This tendency to base decisions largely on the observed decisions of other agents has recently been modelled as information externalities. Banerjee (1992) and Bikhchandani, Hirshleifer and Welch (1992) (henceforth BHW) made the first models which stressed the inefficiencies of these information externalities in a context of social learning ${ }^{2}$. Both models consider a population of individuals each endowed with a private, costless and imperfect signal concerning the desirability of a course of action. People decide sequentially whether to adopt or reject a given course of action. People observe which actions were taken by the persons who moved before them, but they do not observe their signals. If enough individuals have adopted the same behaviour, then each subsequent individual neglects her private signal and herds on the actions of the first persons, because the informativeness of their combined actions is higher than the informativeness of any one signal. More interestingly they also showed that herding is quite likely to cause a "bad outcome", i.e. an outcome where all (or the vast majority of all) players adopt an action which ex post turns out to be suboptimal.

Subsequently a number of other papers appeared which also stressed the inefficiencies entailed by information externalities in different contexts of social learning. For example ${ }^{3}$ Chamley and Gale (1994) consider a set-up similar to BHW except that all players have the possibility to wait in order to observe how many

\footnotetext{
${ }^{1}$ Caminal and Vives (1996) analyse a game where firms engage in price competition in order to become more popular and benefit from these information externalities.

${ }^{2}$ With a "context of social learning" we mean a context where one person must choose an action out of a prespecified action set and which has, prior to her decision, the opportunity to learn out of the choices made by her predecessors.

${ }^{3}$ The models we briefly discuss in this paragraph are among the most famous ones, but the list is certainly not exhaustive.
} 
players invest in the current period and to make their investment decision in the next period based on superior information. They showed how in their context bad outcomes and inefficient waiting may occur in equilibrium. Avery and Zemsky (1998) also consider a set-up similar to BHW except that they add a competitive market maker in the picture which sets the price of an investment asset on the basis of all available information. They show that due to herding short-run price bubbles can occur provided that traders are uncertain about the precision of the other traders' signals. Vives (1993) shows that in the presence of a continuous action space in the long run bad outcomes do not arise, i.e. eventually everyone will adopt the right action. However in the presence of noise (when observing the actions of the other players) the rate of convergence towards the right action is slow.

To summarise, so far the literature on social learning mainly stressed different inefficiencies (short-run price bubbles, bad outcomes, inefficient waiting, slow learning, ...) entailed by information externalities. All these inefficiencies are due to the fact that players when choosing their actions rely too little on their private information and too much on the public one. In this paper we argue that herding ${ }^{4}$ also possesses some positive aspects in the sense that players may have less incentives to wait in a herding environment.

We study the following set-up. We assume that a fixed number of firms enter sequentially in an emerging market. Each firm is run by one manager who maximises his utility. Upon entrance in the market managers must choose a technology. Managers can choose to adopt an "old" technology. Adopting this "old" technology is easy: it doesn't require effort from the manager. Managers can also choose to exert a high effort to check the existence of a new (and more profitable) technology. With a probability p a new technology exists, which will always be found if effort is provided. We assume that - due to switching costs - it's only profitable to innovate upon entrance in the market. After the adoption of the old technology it's only profitable for a firm to imitate the new technology if it is invented by a subsequent manager. Managers act strategically in the sense that they all may wait, adopt the "old" technology in the hope to free-ride on the effort of another manager inventing a better technology.

\footnotetext{
${ }^{4}$ From now on, with "herding" we mean a behaviour where one person observes the action(s) of her predecessor(s), updates her prior beliefs and then has more incentives to imitate her predecessor(s) knowing that her choice may ex post not be optimal. This definition allows us to classify Vives' (1993) paper also in the herding literature and it permits us to better explain the originality of this paper.
} 
We first compute the (unique) equilibrium of our game if everyone observes the effort levels and the technological choices of all their predecessors. In these circumstances there are no informational asymmetries and thus also no herding. In this benchmark case all first movers wait and free-ride on the effort level of the last manager. This benchmark case is thus characterised by a lot of free-riding 5 and, as switching from the old to the new technology is inefficient, note that in our model free-riding is inefficient. This free-riding is driven by our assumption that the new technology is a public good. Once a manager invented the new technology, by assumption it can be copied by all the other managers in the economy at no cost. As inventing the new technology constitutes a costly activity all managers in our model have a natural incentive to wait in order to free-ride on someone else's effort level.

Next we assume that all managers only observe one another's technological choices (and not one another's effort levels). We show how herding attenuates the freeriding problems present in our benchmark case. The intuition goes as follows. Late movers observe the early movers adopting the old technology. As they infer the inexistence of the new technology out of their actions, this induces them to adopt the old technology as well (in other words late movers tend to herd on the actions of the early movers). Early movers realise that they have little possibilities to freeride on the effort level of late movers and this induces them to exert effort. This paper thus highlights the existence of a trade-off between herding and waiting (or free-riding).

We also show that late entrants (who put a lower probability on the existence of the new technology as compared to the one put by early entrants) may exert a higher ex ante effort level than early entrants. This is because in equilibrium the probability with which the second up to the last manager exert effort is determined by the interplay of two effects. The first effect, which we call the herd effect, states that the more managers who adopt the old technology, the higher the probability that a previous manager unsuccessfully checked the existence of a superior technology, the lower a manager's incentives to exert effort. As mentioned in our

\footnotetext{
${ }^{5}$ And with free-riding we mean that a manager takes an inefficient action (i.e. adopts the standard technology) in the hope to free-ride on the effort of another manager. Free-riding and waiting are thus equivalent in our model and henceforth we will almost always denote this behaviour by waiting.
} 
previous paragraph, this effect explains why with unobservable effort levels our players have less incentives to wait. The second effect which we call the free-rider effect states that late entrants know that they cannot rely too much on the effort of a subsequent manager inventing a better technology. This effect ensures that late entrants still face a lot of incentives to exert effort. We show that - depending on the values of our exogenous parameters - the free-rider effect may dominate the herd effect. In that case late entrants exert a higher ex ante effort level than early ones.

In the following paragraphs we discuss more in depth how this paper relates to other models in the literature of social learning. We discuss the other papers in increasing order of relatedness.

Smith and Sørensen (1997) study a set-up similar to the one of BHW (i.e. without analysing issues of strategic waiting) in which they consider a social planner who maximises the present value of all agents' utilities. In their paper a socially more efficient outcome is obtained when some players internalise their information externality. They show that players can be induced to internalise their information externalities by means of a simple set of history-contingent balanced-budget transfers. In our paper information externalities are internalised because all players know that - due to herding - they will be less able to free-ride on the effort levels of subsequent movers.

Rob (1991) models dynamic entry in an emerging market. Potential entrants observe the actions (quantity choices) and the payoffs (profits) of the incumbent firms and out of this infer information concerning the realisation of an unknown demand parameter. Due to an information externality the rate of entry in the emerging market is lower than the socially optimal one. His model does not possess our main insight that herding may act as an incentive device because in his model the act of waiting does not entail any negative information externality. In his model an information externality only occurs whenever a firm enters in the emerging market. If a firm decides to wait (to gather information from the entry decisions of the other firms) this does not influence the remaining players' posteriors concerning the profitability of the market (and thus doesn't hamper their incentives to enter the emerging market at a later date).

In Chamley and Gale (1994) some players receive an option which gives them the 
right to invest. The higher the realised state of the world, the higher the probability that each player receives an investment option and the higher the return from the investment project. All players who possess an investment option have the possibility to wait, to observe how many other players invested in the previous period and to make an investment decision on the basis of more information. They prove the existence of a unique equilibrium where all players who possess an investment option invest with a symmetric probability $\lambda$ such that the informational gain of waiting is offset by the opportunity cost of waiting.

Herding does not reduce the scope of strategic waiting in their model. If in their model all players would truthfully exchange their signals, then first best applies i.e. everyone would take the correct ex ante investment decision in period one. Why is it that in our model herding reduces the scope of strategic waiting and not in theirs? Which crucial assumptions explain this difference? In our model we assume (i) the existence of aggregate uncertainty (in Chamley and Gale's model if the number of players becomes large there is no (or almost no) aggregate uncertainty) and (ii) that the good state of the world becomes publicly known as soon as one player exerts effort. To see that it are really these two assumptions which make the crucial difference, take Chamley and Gale's model but assume that (i) the economy is populated by a limited number of players who possess an imperfect signal and (ii) the return of the investment project becomes publicly known as soon as one player invests ${ }^{6}$. If players were to share truthfully their signals then they would all possess the same posteriors. In this context - due to aggregate uncertainty (due to the limited number of players) - everyone still has an incentive to wait until someone else invests (and thereby resolving the aggregate uncertainty). Assume now that our players do not exchange their signals (due for instance to

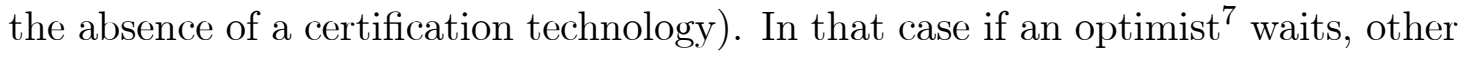
players partly infer a bad signal out of this. As this hampers their future incentives to invest, optimists have then less incentives to wait.

In Hendricks and Kovenock (1989) two oil firms possess a private imperfect signal concerning the profitability of drilling an exploratory oil well. If one firm drills, then the other firm costlessly observes whether there is oil or not and (in case of success) makes a riskless investment decision in the next period. Both firms wait

\footnotetext{
${ }^{6}$ Note that this merely represents the set-up analysed by Hendricks and Kovenock (1989).

${ }^{7}$ In Chamley and Gale, an optimist can be interpreted as a player who possesses an investment option.
} 
in the hope to free-ride on the drilling cost of the other firm. If one firm delays its drilling decision, this "signals" to the other firm that it possesses a poor signal. The subsequent downward revision of its prior may induce the other firm to let its lease expire without drilling any well (which is nothing else than the herd effect we mentioned earlier). Herding does not act as an incentive scheme in their model because they only work with a two-period model. In their model both firms don't care about each other's second-period posteriors because (by construction) they cannot free-ride on each other's second-period drilling decision. This paper thus shows that additional insights can be obtained when generalising their model to $N$ periods.

This paper is organised as follows. In section two we explain the basic assumptions of our model. We first analyse the workings of our model under the assumption of observable effort levels and observable technological choices (section 3). In section 4 we work under the assumption that players do not observe one another's effort levels. First we illustrate how herding acts as an incentive device by focusing on a simple equilibrium where only one manager exerts effort. Next (section 4.2) we analyse the case where $N$ managers exert effort (with a certain probability) in equilibrium. Final comments are summarised in section 5 .

\section{The Model}

We consider a simple model of dynamic entry in an emerging market. Our model counts two phases: a first one called the adoption phase and a second one called the production phase. Throughout the paper time is discrete. In the adoption phase we assume that in each different period $t=1, \ldots, K(K \geq 2(K \in \mathbb{N}))$ one (and only one) firm enters in a market and makes her technological choice. Each firm is run by a risk neutral manager/entrepreneur who maximises an expected utility function. Henceforth we call manager $t$, the manager who enters in the emerging market at time $t$. Next, (after period K) in the production phase all managers receive their payoffs by producing and selling their goods in a finite market (which lasts from period $K+1$ until period $\bar{K}(\bar{K} \geq K+1(\bar{K} \in \mathbb{N})))$.

Manager $t$ must use a technology (chosen out of the set $T_{t} \in\{o, n\}$ ) from his time of entry until time $\bar{K}^{8}$. In the beginning of the first period $T_{1}=\{o\}$. o

\footnotetext{
${ }^{8}$ Stated differently, manager $t$ is not allowed to make his technological choice at time $t^{\prime}>t$.
} 
represents an "old" technology. One can best think of $o$ as a technology which is widely used in other industries, everyone knows how $o$ works and everyone knows its profitability characteristics. Assume that $T_{t}=\{o\}$. Manager $t$, before adopting his technology, has then two options: he can either adopt $o$ at no cost (i.e. without exerting effort) or he can exert effort $e$ to try to invent a new, more profitable technology denoted by $n$. If manager $t$ successfully invents $n$, then $\forall t^{\prime} \geq t, T_{t^{\prime}}$ becomes equal to $\{n\}$ which implies that manager $t$ (along with all his successors) has no other option left than to adopt the new technology. Moreover we assume that once $n$ was invented, all previous managers instantaneously exchange their old technology for the new one and stick to it until the end of the game ${ }^{9}$. If manager $t$ doesn't invent $n$, then $T_{t}$ remains equal to $\{o\}$ and in the beginning of period $t+1, T_{t+1}=\{o\}$. It is assumed that the new technology exists with a probability $\mathrm{p} \in(0,1)$ and will always be invented if it exists and if a manager exerts effort e. We assume that if manager $t$ invents $n$, all previous and subsequent managers benefit from a knowledge spillover effect in the sense that they adopt $n$ without incurring the cost of effort (however previous managers who switch from $o$ to $n$ incur a switching cost (see below)). The use of $n(o)$ during the production phase generates a total profit of $\bar{\pi}(\cdot)(\underline{\pi})$. The "height" of $\bar{\pi}(\cdot)$ depends on whether or not a manager had adopted $o$ prior to his adoption of $n$. Formally, $\bar{\pi}(\cdot)$ is determined by the function $\bar{\pi}: I 1 \longrightarrow \mathbb{R}$ where $I 1=\{s, n s\}$ represents an indicator function which takes the value $s(n s)$, whenever a manager switched from $o$ to $n$ (adopted $n$ from scratch).

We model the managers' utilities as a v.N.M. utility function. Suppose $T_{t}=\{o\}$. If manager $t$ decides to exert effort at time $t$ his expected payoff equals $E(U \mid e, n s)=$ $p \cdot U(\bar{\pi}(n s))+(1-p) \cdot U(\underline{\pi})-e(e>0)$. To hold matters simple, we assume that $U(\underline{\pi})=0$ and that $U(\bar{\pi}(n s))=1$, so $E(U \mid e, n s)=p-e$. If an entrant exerts effort, with probability p there exists a new technology which he will find (for sure) and in the production phase this will give him a payoff equal to $1-e$. However our diligent manager may be unlucky because with probability $(1-p)$ there does not exist a new technology. In that case our manager must use $o$ to operate in the

This assumption could best be defended by allowing all firms to produce and sell their goods from their time of entry on. However, this would imply that a manager's payoff would depend on his time of entry, an unnecessary complication.

${ }^{9}$ This is a model about technology invention and how herding affects the time at which a new technology gets discovered. Therefore we don't explicitly model how a new technology spreads throughout the economy. Instead we exogenously assume that once someone invents $n$, it automatically (and instantaneously) gets adopted by the other managers. 
emerging market. In the production phase he will then get a payoff equal to $-e$. We also assume that - due to switching costs $-U(\bar{\pi}(s))=\gamma<1$. In order to highlight the interesting features of our model, we suppose that:

A1: $p>e$.

Under assumption one all our (risk neutral) managers have an individual incentive to exert effort at their time of entry. We also assume that all managers rather imitate instead of innovate. This is captured by our following assumption:

A2: $p-e<p \gamma$

Assumption A2 states that given $\gamma$, manager one rather waits (and never exerts effort) and free-rides on the effort of the second manager, if he believes the second manager will exert effort for sure. So A2 naturally introduces strategic waiting in our model. If a manager who did not exert effort and adopted $o$ in a previous period decides to exert effort at time $t$, he gets $E(U \mid e, s)=p \gamma-e$. We assume that:

A3: $p \gamma<e$

Assumption 3 is an important simplifying assumption. It ensures that in equilibrium managers only want to innovate at their time of entry. The main difference between our exogenous queue assumption and the one present in Banerjee (1992) and BHW (1992) is that in our model all managers still care about their successors' actions. The precise repercussions of A3 will be discussed more in detail in the next sections.

\section{Observable effort levels and observable tech- nological choices: A game of free-riding.}

In this section we analyse our model under the assumption that all managers observe one another's effort levels and technological choices. The timing of our game is explained below ${ }^{10}$ :

\footnotetext{
${ }^{10}$ The timing of the game is explained under the restriction that managers only exert effort (i) at their time of entry and (ii) if $n$ had not yet been invented. By now it should be clear that these restrictions are innocuous.
} 
0) At time zero: nature decides whether $n$ exists or not.

1) At time $t$ : manager $t$ enters in the market and observes $T_{t}$ and the past effort levels of all his predecessors. In case $T_{t}=\{o\}$, manager $t$ chooses his effort level. If he invents $n, T_{t}$ becomes equal to $n$, the new technology is immediately adopted by manager $t$ and copied by all his predecessors and subsequent followers.

2) Period $K+1$ until period $\bar{K}$ : managers receive their payoffs and the game ends.

We assumed that the new technology will always be invented if it exists and if a manager exerts effort $e$. The inexistence of $n$ is thus proven as soon as a manager exerts effort and adopts $o$. Hence a manager only wants to exert effort (i) at his time of entry, (ii) if none of his predecessors adopted $n$ and (iii) if none of his predecessors exerted effort. Therefore we define a strategy for manager $t, q_{t}$, as a probability with which manager $t$ exerts effort at his time of entry if none of his predecessors exerted effort. A subgame perfect equilibrium (SPE) is a $\left(q_{1}, q_{2}, \ldots, q_{K}\right)$ such that no manager has an incentive to deviate given the other managers' strategies. In this case we obtain the following result:

PROposition 1 Under A1, A2 and A3, with observable technological choices and observable effort levels, the unique SPE of our game is: $q_{1}=q_{2}=\ldots=q_{K-1}=0$, $q_{K}=1$.

Proof: Consider first the optimal decision of manager K. Manager K observes that all his predecessors use the standard technology. But he also observes that none of his predecessors exerted a high effort level. Therefore manager $\mathrm{K}$ knows that if he exerts a high effort level with prior probability $\mathrm{p}$ he will find the superior technology. Under A1, manager $\mathrm{K}$ wants to exert effort. As mentioned previously, under A3 none of the K-1 first movers want to exert effort at time K. Therefore $q_{1}=q_{2}=\ldots=q_{K-1}=0, q_{K}=1$ constitutes an equilibrium strategy. Under A2 the first $K-1$ movers rather wait and free-ride on manager K's effort level. Therefore $q_{1}=q_{2}=\ldots=q_{K-1}=0, q_{K}=1$ constitutes the unique (subgame perfect) equilibrium. Q.E.D.

Note that proposition (1) crucially rests on A3. This can easily be illustrated with an example where $K=2$. If $p \gamma \geq e, q_{1}=1, q_{2}=0$ also constitutes a subgame perfect equilibrium. To see this, suppose that manager one exerts no effort and adopts $o$. Call $q_{12}$ the probability with which manager one exerts effort at time two. In the second period there are two firms in the market with two managers 
who both have an incentive to exert effort. This subgame possesses three Nash equilibria: $q_{12}=1, q_{2}=0 ; q_{12}=0, q_{2}=1$ and there also exists a mixed-strategy Nash equilibrium in which $q_{12}=\frac{p-e}{p \gamma}$ and $q_{2}=\frac{p \gamma-e}{p \gamma}$. If manager one at time one anticipates that if he were to exert no effort, in period two they would either play the mixed-strategy Nash equilibrium or the equilibrium $q_{12}=1, q_{2}=0$, then manager one rather exerts effort in period one. Therefore, if $p \gamma \geq e\left(q_{1}=1, q_{2}=0\right)$ constitutes a SPE. The reader can easily generalise this insight to the case with $\mathrm{K}$ managers, if $p \gamma \geq e$ there are $\mathrm{K}$ different equilibria with each time a different manager who exerts effort.

Proposition (1) shows that if all players have a lot of information at their disposal, this will result in a lot of waiting. This result bears some close resemblance to the one that was derived earlier by Rob(1991). Rob also models dynamic entry in an emerging market under the assumption that all players observe all the incumbents' payoffs and actions. As mentioned in the introduction in his model due to an information externality the rate of entry is lower than the socially optimal one (in other words the equilibrium (just as in our model) is characterised by too much waiting). In the remainder of the paper we will introduce herding in the model (i.e. managers who "blindly" imitate the technological choices of their predecessors in the hope that they exerted effort) by allowing for informational asymmetries. We will see that in a herding environment our players have much less incentives to wait.

In this paper it's natural to take the case without any private information as our benchmark one because of its sharp contrast with our non-benchmark case. Two remarks. First, note that if in Banerjee (1992) or BHW (1992) all players would observe one another's signals and actions, all players would ex ante take the most efficient action and first best would apply. So the choice of our benchmark case makes it explicitly clear that herding only reduces the scope of strategic waiting when in the absence of any private information, players would still have an incentive to delay their (informative) actions. Second we could have chosen the case where all players don't observe actions (i.e. technological choices) nor effort levels during the adoption phase as our benchmark one. We expect that giving all players the possibility to observe one another's technological choices during the adoption phase, this should increase welfare and efficiency (compared to the case where all players don't observe anything in the adoption phase) because late movers would then have the possibility to free-ride on the effort level of an early mover. However, this result is not original nor surprising. Already in Banerjee (1992) and BHW 
(1992) late movers are better off (in an ex ante sense) when they observe their predecessors actions (as compared to the case where they don't observe anything) because they may act with more information than their predecessors.

\section{A waiting game with observable technological choices and unobservable effort levels.}

In this section we consider the same game as the one we analysed in our previous section except that now our players only observe one another's technological choices and not one another's effort levels. A manager is called active if in equilibrium he exerts effort with a strictly positive probability. In this case we define a strategy for manager $t, q_{t}$, as a probability with which manager $t$ exerts effort at his time of entry if none of his predecessors adopted $n$ (in all the other cases a manager strictly prefers not to exert effort). As effort levels are unobservable this is a dynamic game of imperfect information. Each manager - upon observing his predecessors adopting the old technology - must have a belief concerning which node in the game tree was reached. If a manager believes that at least one of his predecessors exerted effort, then he rather adopts the old technology without exerting effort. In this model beliefs concerning past effort levels matter because they ultimately influence $p_{t}=\operatorname{Prob}[n$ exists $\mid t-1$ first managers use $o]$, which in its turn influences manager $t$ 's incentives to exert effort. Therefore we define our equilibrium concept using each manager's posterior (concerning the existence of the new technology) instead of working directly with his beliefs concerning past effort levels. $P=\left(p, p_{2}, \ldots, p_{K}\right), Q=\left(q_{1}, q_{2}, \ldots, q_{K}\right)$ and $Q_{-t}=\left(q_{1}, q_{2}, \ldots, q_{t-1}, q_{t+1}, \ldots, q_{K}\right)$. A subgame perfect equilibrium (SPE) is a $(Q, P)$ such that:

(i) given $p_{t}$ and $Q_{-t}$, manager $t$ cannot gain by deviating $\forall t$, and

(ii) given $Q, p_{t}$ is computed via Bayes' law, $\forall t$.

We now analyse equilibrium strategies in our game. We first focus on an equilibrium with one active manager which already shows how in this model herding acts as an incentive device. Next we generalise our results to $N(1<N \leq K)$ active managers.

\subsection{An equilibrium with one active manager.}

Our main result with one active manager is summarised below: 
PROposition 2 Under A1 and A2, there exists a SPE in which only the first manager is active and where $q_{1}=1$.

Proof: Suppose all managers revise their priors under the assumption that $q_{1}=1$, $q_{2}=q_{3}=\ldots=q_{K}=0$. It is then quite easy to see that under this assumption it's optimal for manager one to exert effort with probability one. Suppose manager one deviates exerts no effort and adopts $o$. Manager two at his time of entry observes that manager one adopted $o$. His expected gain of exerting effort equals: $P(\mathrm{n}$ exists $\mid$ man. 1 uses $o)-e=p_{2}-e$, where:

$$
p_{2}=\frac{\left(1-q_{1}\right)}{\left(1-q_{1}\right)+q_{1}(1-p)} p
$$

Manager two computes $p_{2}$ by replacing $q_{1}$ in (1) by 1 . Therefore $p_{2}=0$. This is logical: manager two is sure that manager one exerted effort. Therefore he interprets manager one's act of adopting $o$ as a "proof" that $n$ does not exist. Since $p_{2}=0<e, q_{2}=0$. Manager three observes that the first two managers adopted $o$. Using Bayes' law he computes his posterior $p_{3}=\mathrm{P}(n$ exists $\mid$ first two man. use $o$ ):

$$
p_{3}=\frac{\left(1-q_{1}\right)\left(1-q_{2}\right)}{\left(1-q_{1}\right)\left(1-q_{2}\right)+\left[1-\left(1-q_{1}\right)\left(1-q_{2}\right)\right](1-p)} p
$$

in which he substitutes $q_{1}$ by one and $q_{2}$ by zero. Hence $p_{3}=0<e$ and consequently $q_{3}=0$. All subsequent movers compute the same posterior $\left(p_{4}=\right.$ $p_{5}=\ldots=p_{K}=0$ ) and therefore it's optimal for them not to exert any effort $\left(q_{4}=q_{5}=\ldots=q_{K}=0\right)$. Manager one knows this. Correctly anticipating that $q_{2}=q_{3}=\ldots=q_{K}=0$, manager one knows that he is the only one for whom it is profitable to exert effort. Hence it is optimal for him to exert effort with probability one.

Of course this is not the unique equilibrium with one active manager. For example there also exists a SPE in which only the second manger is active and where $q_{2}=1$ (under A2 it is indeed optimal for manager one to set $q_{1}=0$ and to free-ride on the effort level of the second manager). As a matter of fact with only one active manager there exist $\mathrm{K}$ equilibria with each time a different manager who exerts effort for sure, but none of them entail more waiting than the (unique) equilibrium we obtained when effort levels were observable. Q.E.D. 
Intuitively, the strategy in which only the first manager exerts effort for sure constitutes a SPE because manager one - knowing that if he adopts $o$, subsequent movers will revise downward their prior probabilities and will never exert effort internalises his information externality by exerting effort. Therefore in equilibrium one obtains less waiting. Note also that proposition (2) does not rely at all on A3, even if $\gamma$ were equal to one, the strategies described in the proposition would still constitute a SPE.

\subsection{An equilibrium with $\mathbf{N}(1<N \leq K)$ active managers.}

\subsubsection{An existence and uniqueness theorem.}

With $N$ managers, all managers compute their equilibrium probabilities out of the following set of $N+(N-1)$ nonlinear simultaneous equations. The first $N$ equations merely state that our $N$ managers must choose their effort levels such that everyone, given their posteriors, is indifferent between the two pure strategies:

$$
\begin{gathered}
p-e=q_{2} p \gamma+\left(1-q_{2}\right) q_{3} p \gamma+\ldots+\prod_{k=2}^{N-1}\left(1-q_{k}\right) q_{N} p \gamma \\
p_{2}-e=q_{3} p_{2} \gamma+\left(1-q_{3}\right) q_{4} p_{2} \gamma+\ldots+\prod_{k=3}^{N-1}\left(1-q_{k}\right) q_{N} p_{2} \gamma \\
\cdot \\
\cdot \\
\cdot \\
p_{N-1}-e=q_{N} p_{N-1} \gamma \\
p_{N}=e
\end{gathered}
$$

The remaining $(N-1)$ equations determine the posteriors of manager two to manager $N$ (since $p_{1}=p$, the "posterior" of the first manager is exogenously given and need not be endogenously computed). These equations can be summarized as:

$$
p_{j}=\frac{\prod_{k=1}^{j-1}\left(1-q_{k}\right)}{\prod_{k=1}^{j-1}\left(1-q_{k}\right) p+(1-p)} p \quad \forall j=2, \ldots, N
$$

We can now state our most interesting finding:

PROPOSITION 3 Under A1, A2 and if $\gamma \leq \operatorname{Max}\left\{\frac{e}{p}, 1-e\right\}$, for every set of managers, there exists a unique SPE where they are all active. 
Proof: see appendix one and two

Our game thus clearly exhibits multiple equilibria in the sense that different sets of active managers lead to different SPE. ${ }^{11}$ This multiplicity does not bother us in the sense that no equilibrium of our game with non-observable effort levels entails more waiting than the unique equilibrium we obtained with observable effort levels.

Before explaining the intuition behind proposition (3) more in detail, we first illustrate it with a simple example with two active managers

\subsubsection{An illustration with two active managers.}

Assume it are the first two managers who must be active. Both managers compute their equilibrium probabilities out of the equations:

$$
\begin{gathered}
p-e=q_{2} p \gamma \\
p_{2}=e
\end{gathered}
$$

where $p_{2}$ is determined by equation (1). Out of (3), we see that $q_{2}=\frac{p-e}{p \gamma} \in(0,1)$. Out of $(1)$, one can easily see that $q_{1}=\frac{p-e}{p(1-e)} \in(0,1)$. Note that $q_{2}$ is a decreasing function of $\gamma$. This is logical: manager one is only indifferent between innovation and imitation if every increase in the switching cost (decrease in $\gamma$ ) is compensated by an increase in $q_{2}$. If $\gamma$ is low enough (lower than $1-e$ ) this implies that manager two in equilibrium exerts a higher (ex ante) effort level (which is quite counterintuitive given that $\left.p_{2}<p\right)$. To prove that $q_{1}=\frac{p-e}{p(1-e)}, q_{2}=\frac{p-e}{p \gamma}$, $q_{3}=q_{4}=\ldots=q_{K}=0$ constitutes a SPE we still must check that no manager (not even the first two managers) has an incentive to exert effort after observing manager two adopting $o$.

First assume that $p \gamma \leq e$. Under this assumption the first two managers don't want to exert effort from date three on. Manager three computes his posterior using (2) in which he substitutes $q_{1}$ and $q_{2}$ by their equilibrium values. As $q_{2}>0$, $p_{3}<p_{2}=e$. Hence $q_{3}=0$. As previously, $p_{4}=p_{5}=\ldots=p_{K}=p_{3}$ and $q_{4}=q_{5}=\ldots=q_{K}=0$. So the third up to the last manager always adopt the same technology as the one which was adopted by manager two. The first two

\footnotetext{
${ }^{11}$ Specifying a model with an endogenous queue should not limit the number of equilibria. Admittedly, an endogenous queue should yield a unique symmetric equilibrium, but should also possess a large number of asymmetric equilibria.
} 
managers - even though they both prefer to imitate instead of innovate - correctly anticipate that they won't be able to free-ride on the effort levels of subsequent movers. Therefore they are indifferent between the two pure strategies and they may as well choose to exert effort with a strictly positive probability. Hence, if $p \gamma \leq e$, there exists a unique SPE in which only the first two managers are active.

However, even if $p \gamma>e$, there may still exist a SPE in which only the first two managers are active and exert effort with probabilities $q_{1}=\frac{p-e}{p(1-e)}$ and $q_{2}=\frac{p-e}{p \gamma}$. To see this suppose that $\frac{e}{p}<\gamma \leq 1-e$. We know that if $\gamma \leq 1-e, q_{2} \geq q_{1}$. Suppose both managers don't exert effort and adopt the standard technology. Consider both managers in the third period. What induces them to exert effort (given that they know their own effort levels)? Nothing! Manager one observed that the second manager adopted the standard technology. In the third period manager one computes his posterior $p_{13}=\frac{\left(1-q_{2}\right) p}{\left(1-q_{2}\right)+q_{2}(1-p)}$. Now $p_{13} \leq e$ (and thus $p_{13} \gamma<e$. This is logical: manager one's posterior (at time three) is not higher than the one of manager two (who was already indifferent) because $q_{2} \geq q_{1}$. Hence manager one, upon observing manager two adopting the standard technology has no incentive anymore to exert effort. Manager two doesn't want to exert effort in any subsequent period either because $p_{2}=e$. Hence if $\gamma \leq 1-e$ both managers know that they won't be able to free-ride on the effort level of a manager (including their own effort level) after the second period. Given that they are indifferent, they may as well choose to exert effort with a strictly positive probability.

Note that the third up to the last manager never exert effort and adopt the same technology as the one which was adopted by the first two managers. Note that this herd may be misdirected because with a probability $\left(1-q_{1}\right)\left(1-q_{2}\right) p$ all firms in the emerging market end up using the old technology when a new one exists, but which was not invented by a diligent manager. This explanation of how an industry can get "stuck" with a suboptimal technology was already offered by Hendricks and Kovenock (1989). J.Zwiebel (1995) explains this observation on the basis of reputational concerns.

\subsubsection{Herd effect versus free-rider effect.}

In these paragraphs we provide an answer to questions like: what is the intuition behind proposition (3) and which "forces" influence the equilibrium effort level of each active manager? 
Before answering these questions we first introduce some new notations. $z_{t}$ denotes the probability with which manager $t$ will not exert effort (i.e. $z_{t}=1-q_{t}$ ). $j \in \mathbb{N}$ and $j=2, \ldots, N . Z_{j+1}$ denotes the probability that no manager moving after manager $\mathrm{j}$ will search for the new technology (i.e. $\forall j=2, \ldots, N-1$, $Z_{j+1}=z_{j+1} . z_{j+2} \ldots z_{N}$ and if $\left.j=N, Z_{N+1}=1\right)$. Furthermore we define $N E_{j-1}$ as $p / p_{j}$. In other words $N E_{j-1}$ denotes the negative externality induced by the actions of the $j-1$ first managers on manager j's posterior. $N E_{j-1}>1 \forall j$ and $N E_{j-1}$ is strictly increasing in $j$. We define Roe (return on effort) as $p / e$.

All active managers must be indifferent between the two pure strategies, i.e. $p_{j-1}-$ $e=\left(1-Z_{j}\right) p_{j-1} \gamma$. We can rewrite this last equation as:

$$
z_{j}=\frac{1}{Z_{j+1}}\left[C+\frac{N E_{j-2}}{R o e \gamma}\right]
$$

(where $C=\left(1-\frac{1}{\gamma}\right)$ and $\left.N E_{2-2}=N E_{0}=1\right)$ Equation (4) shows that $q_{j}$ is a function of two opposing effects (or "forces").

The first effect, which we call the herd effect, is captured by the term $N E_{j-2}$. $N E_{j-2}$ is increasing in $j$ and $q_{j}$ is decreasing in $N E_{j-2}$ : the more one advances in the queue, the higher the sum of the expected efforts spent by all previous managers, the lower the posterior of manager $j-1$, the lower the probability with which manager $j$ must exert a high effort to make manager $j-1$ indifferent (ceteris paribus). This herd effect is similar to the one present in the other herding models (Banerjee (1992), BHW(1992), Chamley and Gale (1994), Vives (1993), Hendricks and Kovenock (1989), Scharfstein and Stein (1990), ...). However in this model, due to the herd effect, all $N$ managers realise that they won't be able to free-ride too much on the effort levels of their successors. Proposition (3) then shows that by appropriately choosing all effort probabilities one can make all $N$ managers indifferent between the two pure strategies and thus willing to randomise. Therefore our model highlights a trade-off between herding versus waiting which went unnoticed in the current herding literature. The fundamental reason why this insight is not present in Rob (1991), Chamley and Gale (1994) and Hendricks and Kovenock (1989) was already explained in the introduction.

In our model the herd effect also gives birth to a countervailing force which we call the free-rider effect. This free-rider effect is captured by the term $Z_{j+1} \cdot Z_{j+1}$ is increasing in $j$, and $q_{j}$ is increasing in $Z_{j+1}$. This is also logical: the more one 
advances in the queue, the lower the probability that manager $j-1$ can hope to free-ride on the effort of a subsequent manager (due to the herd effect), the higher the probability with which manager $j$ should exert a high effort to make manager $j-1$ indifferent. This free-rider effect thus ensures that late movers may still face a lot of incentives to exert effort.

Equation (4) also shows that manager $j$ 's equilibrium behaviour can be summarised by the following rule: "manager $j$ takes $p_{j-1}$ and $Z_{j+1}$ as given and chooses $q_{j}$ such that manager $j-1$ is indifferent between the two pure strategies". ${ }^{12}$ This simple rule also provides a partial intuition why, once we fix the set of active managers, there exists a unique vector of equilibrium probabilities. $Z_{j+1}$ is fixed such that manager $\mathrm{j}$ is indifferent. Since $q_{j-1}>0$ (otherwise manager $j-1$ is not active), $p_{j-1}>p_{j}$. Therefore if $q_{j}=0$, manager $j-1$ rather exerts effort, if $q_{j}=1$, manager $j-1$ rather waits. By continuity, there exists a unique $q_{j}$ such that manager $j-1$ is indifferent.

One final word of explanation concerning our assumption A3. As explained above, proposition (3) does not fully rely on A3: for high enough a $1-e$, that proposition remains valid even with "low" or "moderate" switching costs. However, the main insight of this paper is that a herding environment may be less prone to problems of strategic waiting than a non-herding one. This was shown by comparing proposition (3) to proposition (1). As proposition (1) crucially rests on A3, our main insight crucially hinges on this assumption. Nonetheless we believe our main insight to be robust in the sense that we can specify two other realistic contexts in which our main insight remains valid and would not depend on A3. First, we can work in a context where $\gamma=1$, where the queue is endogenous and where all players face a discount factor $\delta<1$. Second, in the introduction we already mentioned that our main insight can be recovered without the use of A3 in Chamley and Gale's model provided that we allow for (i) aggregate uncertainty and (ii) the state of the world which becomes publicly known as soon as one player invests.

\footnotetext{
${ }^{12}$ The intuition behind manager one's behaviour is more complicated and is not explained in this paper. Somewhat surprisingly it turns out that manager one chooses $q_{1}$ such that manager $N$ is indifferent. The intuition why manager one is able to make the last manager indifferent was explained in a previous version of this paper titled "Corporate Conservatism as Endogenous Pessimism" and can be obtained by simple request from the author. For a mathematical explanation see proposition (6) in appendix one.
} 


\subsubsection{The temporal profile of equilibrium effort probabilities.}

In this subsection we check when the free-rider effect dominates the herd effect (i.e. when late movers exert a higher ex ante effort level than early movers).

PROPOSITION 4 In equilibrium: $q_{1} \leq q_{2} \leq \ldots \leq q_{N}$ if and only if $\gamma \leq 1-e$, and $q_{1}>q_{2}>\ldots>q_{N}$ if and only if $\gamma>1-e$

Proof:

From appendix one we know that we can rewrite our system of $N+(N-1)$ simultaneous equations into a simpler set of $N$ equations in $N$ unknowns:

$$
\begin{gathered}
\kappa_{2}+\gamma z_{2} z_{3} \ldots z_{N}=\kappa_{1} \\
\kappa_{2} z_{1}+\gamma z_{1} z_{3} \ldots z_{N}=\kappa_{1} \\
\kappa_{2} z_{1} z_{2}+\gamma z_{1} z_{2} z_{4} \ldots z_{N}=\kappa_{1} \\
\vdots \\
\kappa_{2} z_{1} \ldots z_{N-1}+\gamma z_{1} \ldots z_{N-1}=\kappa_{1}
\end{gathered}
$$

where $\kappa_{2}=1-\gamma-e \in(-1,+1), \kappa_{1}=e \frac{1-p}{p} \in(0, \infty)$

Assume that $\gamma \leq 1-e$. Then the first terms on the LHS of the equations form a decreasing sequence $\left(\kappa_{2} \geq \kappa_{2} z_{1} \geq \ldots \geq \kappa_{2} z_{1} \ldots z_{N-1}\right)$, so the second terms on the LHS must form an increasing sequence: $z_{2} z_{3} \ldots z_{N} \leq z_{1} z_{3} z_{4} \ldots z_{N} \leq z_{1} z_{2} z_{4} z_{5} \ldots z_{N} \leq$ $\ldots \leq z_{1} \ldots z_{N-1}$. Simplifying these inequalities we get: $z_{1} \geq z_{2}, z_{2} \geq z_{3}, \ldots$, $z_{N-1} \geq z_{N}$ implying that $q_{1} \leq q_{2} \leq \ldots \leq q_{N}$ which is our result. The proof for $\gamma>1-e$ is similar except that the first term of the LHS now forms a strictly increasing sequence and therefore: $q_{1}>q_{2}>\ldots>q_{N}$.Q.E.D.

The intuition behind proposition (4) was already set forth in an earlier part of this paper. With two active managers we saw that if $\gamma$ is relatively low, manager one was only indifferent between the two pure strategies if the high switching cost was compensated by a higher $q_{2}$. For low enough a $\gamma, q_{1}<q_{2}$. Proposition (4) shows that this result can be generalised to the case with $N$ active manager.

We believe proposition (4) to be an interesting one because it proves a quite counterintuitive result that late movers (who put a lower probability on the existence of $n$ than early movers) may - depending on the values of the parameters - exert higher ex ante effort levels than early movers. 


\section{Conclusions}

This paper analysed a waiting game with information externalities. We differed from other papers which introduced information externalities in waiting games (see in particular Chamley and Gale (1994) and Gul and Lundholm (1995)) in two important aspects. First we assumed the existence of aggregate uncertainty and second, we assumed that the good state of the world becomes (publicly) known as soon as one manager exerts effort. In the absence of informational asymmetries the equilibrium exhibits a lot of strategic waiting because all players wait until the last manager exerts effort and resolves the aggregate uncertainty. With unobservable effort levels all managers (except the first one) revise downward their priors because they infer out of the conservative behaviour of their predecessors the unavailability of the new technology. As all players (except the first one) revise downward their prior probabilities, this hampers their incentives to innovate. Early movers correctly anticipate that if they adopt the old technology, they will be less able to free-ride on the effort levels of one of their successors and this induces them to exert effort. Therefore this paper shows that in the presence of aggregate uncertainty and if the good state of the world becomes publicly known as soon as one player invests, there exists a trade-off between herding and waiting which has not been stressed before by other models which introduced informational externalities in waiting games (see a.o. Rob (1991), Chamley and Gale (1994), Gul and Lundholm (1995), Hendricks and Kovenock (1989), ...). Finally, we also showed that if switching costs are relatively high late movers exert a higher ex ante effort level than early movers. We believe this last result to be counterintuitive in the sense that early movers put a higher probability on the existence of the new technology as compared to the one put by late movers.

This paper analysed strategic waiting under the assumption that managers only want to innovate at their time of entry. One may want to relax that assumption and work instead with an endogenous-queue model. We believe this constitutes an interesting topic for future research.

As we mentioned in our introduction, the context in our paper is most realistic if one considers the oil exploration industry. Our framework can also be applied to the timing of compatibility decisions between two technologies: a firm selling technology A may be induced to search for a technology which would make technology $\mathrm{A}$ and $\mathrm{B}$ mutually compatible because by not searching she may discourage the 
other firm from doing so. In our opinion, our theory may be applied to other economic fields as well. For instance the hold-up problem (or more generally problems due to moral hazard in teams) may become less severe in a herding environment because individuals realise that if they don't work enough this may reveal some bad information and this will discourage others from working hard too. In that case our theory can also be applied to the field of (complete and incomplete) contract theory. Similarly, organisations may structure themselves such as to introduce information externalities in their organisations which may induce some people to work. We believe all this to constitute an avenue for future research. 


\section{References:}

Avery, Christopher and Zemsky, Peter "Multi-Dimensional Uncertainty and Herd Behavior in Financial Markets" American Economic Review, September 1998, 88(4), 724-748

Banerjee, Abhijit "A Simple Model of Herd Behavior" The Quarterly Journal of Economics, August 1992, 57(3), 797-817

Bikhchandani, Sushil, Hirshleifer, David and Welch, Ivo "A Theory of Fads, Fashion, Custom, and Cultural Change as Informational Cascades" Journal of Political Economy, October 1992, 100(5), 992-1026

Caminal, Ramon and Vives, Xavier "Why market shares matter: an informationbased theory" Rand Journal of Economics, Summer 1996, 27(2), 221-239

Chamley, Christophe and Gale, Douglas "Information Revelation and Strategic delay in a Model of Investment" Econometrica, September 1994, 62(5), 10651085

Gul, Faruk and Lundholm, Russel "Endogenous Timing and the Clustering of Agents' Decisions" Journal of Political Economy, 1995, 103(5), 1039-1066

Hendricks, Kenneth and Kovenock, Dan "Asymmetric Information, Information Externalities, and Efficiency: the Case of Oil Exploration" Rand Journal of Economics, Summer 1989, 20(2), 164-182

Rob, Rafael "Learning and Capacity Expansion under Demand Uncertainty" Review of Economic Studies, July 1991, 58(4), 655-675

Scharfstein, David and Stein, Jeremy "Herd Behavior and Investment" American Economic Review, June 1990, 80(3), 465-479

Smith, Lones and Sørensen, Peter "Informational Herding and Optimal Experimentation" mimeo, October 1997

Vives, Xavier "How fast do rational agents learn?" Review of Economic Studies, April 1993, 60(2), 329-347

Zwiebel, Jeffrey "Corporate Conservatism and Relative Compensation" Journal of Political Economy, 1995, 103(1), 1-25 


\section{Appendix one}

by N.Melissas and M.Pauly

In this appendix we prove our existence and uniqueness theorem as stated in proposition (3). We work here under the assumption that managers only exert effort at their time of entry. In appendix 2 we prove that if $\gamma \leq \operatorname{Max}\left\{\frac{e}{p}, 1-e\right\}$ in equilibrium managers only want to exert effort at their time of entry. We start by reducing our system of $N+(N-1)$ simultaneous equations into a more tractable set of $N$ equations in $N$ unknowns. To illustrate our way of working, consider the first equation: $p-e=q_{2} p \gamma+z_{2} q_{3} p \gamma+z_{2} z_{3} q_{4} p \gamma+\ldots+z_{2} \ldots z_{N-1} q_{N} p \gamma$ (where $\left.z_{t}=1-q_{t}\right)$. This equation can be rewritten as: $p-e=\left(1-z_{2} z_{3} \ldots z_{N}\right) p \gamma$. This last equation can be rewritten as: $\kappa_{2}+\gamma z_{2} z_{3} \ldots z_{N}=\kappa_{1}$ where $\kappa_{2}=1-\gamma-e$ and $\kappa_{1}=e \frac{1-p}{p}$.

Similarly we can rewrite equation $t$ as: $(1-\gamma)+\gamma z_{t+1} \ldots z_{N}=\frac{e}{p_{t}}$ (if $t=N$ then $z_{N+1}=1$ ). Replacing $\frac{1}{p_{t}}$ by $1+\frac{c}{z_{1} \ldots z_{t-1}}$ (where $c=\frac{1-p}{p}$ ) in this last equation we get $\kappa_{2} z_{1} \ldots z_{t-1}+\gamma z_{1} \ldots z_{t-1} z_{t+1} \ldots z_{N}=\kappa_{1}$. We thus obtain the following set of $N$ simultaneous equations:

$$
\begin{gathered}
\kappa_{2}+\gamma z_{2} z_{3} \ldots z_{N}=\kappa_{1} \\
\kappa_{2} z_{1}+\gamma z_{1} z_{3} \ldots z_{N}=\kappa_{1} \\
\kappa_{2} z_{1} z_{2}+\gamma z_{1} z_{2} z_{4} \ldots z_{N}=\kappa_{1} \\
\cdot \\
\cdot \\
\cdot \\
\kappa_{2} z_{1} \ldots z_{N-2}+\gamma z_{1} \ldots z_{N-2} z_{N}=\kappa_{1} \\
\kappa_{2} z_{1} \ldots z_{N-1}+\gamma z_{1} \ldots z_{N-1}=\kappa_{1}
\end{gathered}
$$

definition: We say that $\left(z_{1}, \ldots, z_{N}\right)$ is an equilibrium if $z_{t}$, computed out of our system of $N$ simultaneous equations, $\in(0,1) \forall t$

We first state and prove our Uniqueness Theorem.

PROPOSITION 5 (Uniqueness Theorem) There cannot be more than one equilibrium. 
Proof:

Suppose there are two equilibria $\left(z_{1}, \ldots, z_{N}\right)$ and $\left(z_{1}^{\prime}, \ldots, z_{N}^{\prime}\right)$. Assume that $z_{1}^{\prime}>z_{1}$. Out of the first equation we know that $z_{2}^{\prime} \ldots z_{N}^{\prime}=z_{2} \ldots z_{N}=\frac{\kappa_{1}-\kappa_{2}}{\gamma}$. Out of the second equation we know that $\kappa_{2} z_{1}+\gamma \frac{z_{1} z_{2} \ldots z_{N}}{z_{2}}=\kappa_{1}=\kappa_{2} z_{1}^{\prime}+\gamma \frac{z_{1}^{\prime} z_{2}^{\prime} \ldots z_{N}^{\prime}}{z_{2}^{\prime}}$. It follows that:

$$
z_{2}=\frac{\gamma z_{1}}{\kappa_{1}-\kappa_{2} z_{1}}\left(z_{2} \ldots z_{N}\right)
$$

Hence, $z_{2}^{\prime}>z_{2}$. Similarly, we learn from equation $t$ that $z_{t}^{\prime}>z_{t}$. But this contradicts that $z_{2}^{\prime} \ldots z_{N}^{\prime}=z_{2} \ldots z_{N}=\frac{\kappa_{1}-\kappa_{2}}{\gamma}$. If $z_{1}^{\prime}=z_{1}$, it's easy to show that $\forall t z_{t}^{\prime}=z_{t}$ and that both equilibria are equal.Q.E.D.

Definition: $\left(z_{1}, \ldots, z_{N}\right)$ is a candidate equilibrium if $z_{t} \in(0,1] \forall t$ and,

$$
\begin{gathered}
\kappa_{2} z_{1}+\gamma a \frac{z_{1}}{z_{2}}=\kappa_{1} \\
\kappa_{2} z_{1} z_{2}+\gamma a \frac{z_{1}}{z_{3}}=\kappa_{1} \\
\vdots \\
\kappa_{2} z_{1} \ldots z_{N-1}+\gamma a \frac{z_{1}}{z_{N}}=\kappa_{1}
\end{gathered}
$$

where $a=\frac{\kappa_{1}-\kappa_{2}}{\gamma}$.

Note that the system of equations starting from (8) to (and including) (9) merely represent a rewriting of the system of equations starting from (6) to (and including) (7). Note also that equation (5) does not intervene in our definiton of our candidate equilibrium. Therefore, every equilibrium is a candidate-equilibrium (to see this, replace (5) by $z_{2} z_{3} \ldots z_{N}=a=\frac{\kappa_{1}-\kappa_{2}}{\gamma}$ and insert it in all the subsequent equations). However, every candidate equilibrium constitutes an equilibrium only if:

(a) all the $z_{t} \neq 1$,

(b) $z_{2} z_{3} \ldots z_{N}=\frac{\kappa_{1}-\kappa_{2}}{\gamma}$

Our next proposition shows that once we know $z_{1}$, we are able to compute all the remaining $z_{j}^{\prime} s(j=2, \ldots, N)$.

PROpOSITION $6 \forall \alpha \in(0,1]$, there exists one and only one candidate equilibrium such that $z_{1}=\alpha$. 
Proof:

If $z_{1}=\alpha$, then equation (8) gives:

$$
z_{2}=\frac{\left(\kappa_{1}-\kappa_{2}\right) \alpha}{\kappa_{1}-\kappa_{2} \alpha}
$$

Note that $\kappa_{1}-\kappa_{2}>0$, because of A2. Therefore $z_{2} \in(0,1]$. We can see from equations (8) to (and including) equation (9) that $z_{j} \in(0,1] \forall j$. Q.E.D.

We know enough now to state our last proposition.

PRoposition 7 (Existence Theorem) Under A1, A2 and A3, $\forall N>1$ there exists an equilibrium vector $\left(z_{1}, \ldots, z_{N}\right)$.

Proof:

Assume that $z_{1}=1$. Then out of our system of equations starting from (8) to (and including) (9) we know that $z_{2}=z_{3}=\ldots=z_{N}=1$ (because $\kappa_{2}+\gamma a=\kappa_{1}$ ). But then $z_{2} \ldots z_{N}=1>\frac{\kappa_{1}-\kappa_{2}}{\gamma}$ (the reader can easily verify that under A1: $\kappa_{1}-\kappa_{2}<\gamma$ ).

Assume now that $z_{1}<\epsilon$ (where $\epsilon$ represents an arbitrarily small strictly positive number). From (8) we know that:

$$
\lim _{z_{1} \rightarrow 0} z_{2}=\lim _{z_{1} \rightarrow 0} \frac{\left(\kappa_{1}-\kappa_{2}\right) z_{1}}{\kappa_{1}-\kappa_{2} z_{1}}=0
$$

Hence $\exists \epsilon_{2}>0$ such that $\forall z_{1} \leq \epsilon_{2}, z_{2}<a^{\frac{1}{N-1}}$

More generally,

$$
\lim _{z_{1} \rightarrow 0} z_{j}=\lim _{z_{1} \rightarrow 0} \frac{\left(\kappa_{1}-\kappa_{2}\right) z_{1}}{\kappa_{1}-\kappa_{2} z_{1} \ldots z_{j-1}}=0
$$

Hence $\forall j, \exists \epsilon_{j}>0$ such that $\forall z_{1} \leq \epsilon_{j}, z_{j}<a^{\frac{1}{N-1}}$.

Let's define:

$$
\epsilon=\min _{j}\left\{\epsilon_{j}\right\}>0
$$

Then $\forall z_{1} \leq \epsilon, z_{j}<a^{\frac{1}{N-1}} \forall j$.

In that case,$z_{2} \ldots z_{N}<a$.

We know that the function $f: z_{1} \in(0,1) \rightarrow z_{2} \ldots z_{N} \in \Re$ is a continuous one because $\forall j, z_{j}$ is a continuous function of $z_{1}$ and we know that the product of continuous functions also yields a continuous function. We also know that $f(1)>a$ and $f(\epsilon)<a$. Hence there exists at least one $z_{1} \in(0,1)$ such that $f\left(z_{1}\right)=a$. Q.E.D. 


\section{Appendix two}

In this appendix we prove that if (sufficient condition) $\gamma \leq \operatorname{Max}\left\{\frac{e}{p}, 1-e\right\}$ all active managers only want to exert efort at their time of entry. If $p \gamma<e$, then by assumption each manager only wants to exert effort at his time of entry. If $p \gamma \geq e$, then we must check whether an active manager has no incentive to exert no effort at his time of entry, to wait untill some of (or possibly all) his (active) succesors made their technological choices and to exert effort at a future date. To analyse this kind of strategic behaviour we study the following variant of our game.

0) At time zero, nature decides whether $n$ exists or not.

1) Manager one enters in the market and chooses his effort level. He only adopts $o$ in case he doesn't invent $n$.

2) Manager two enters in the market and observes the technological choice and the past effort level of the first manager. In case manager one adopted $o$, the first two managers choose simultaneously their effort levels. If one of them invents $n$, it's immediately copied by the other one.

3) In each subsequent period (untill period K), one additional manager enters in the market. In case all previous managers adopted $o$, all managers present in the market choose simultaneously their effort levels. If a manager invents $n$, it's immediately adopted by him and copied by all his predecessors and subsequent followers.

4) In period $K+1$ no manager enters in the market. In case all managers adopted $o$, all active managers choose simultaneously their effort levels. In case $n$ gets invented, it automatically spreads throughout the economy.

5) In period $K+2$ untill period $\bar{K}(\bar{K} \geq K+2)$ : managers receive their payoffs and the game ends.

Assume that $p \gamma=e$. Assume it are the first $N$ managers who are active. As $q_{1}>0$ (otherwise manager one wouldn't be active) $p_{j} \gamma<e(j=2, \ldots, N)$. Assume manager one did not exert effort at time one and assume that the second one adopts $o$. At time three manager one strictly prefers to exert no effort because, as $q_{2}>0$, $p_{13} \gamma<e$. Hence at time two manager one has two options: (i) exert effort at time two (which yields a payoff equal to $p \gamma-e=0$ ) or (ii) never exert any effort untill the end of the game. As $0<\left(1-Z_{2}\right) p \gamma$, manager one only wants to exert effort at time 1 .

Assume now that $\frac{e}{p}<\gamma \leq 1-e . l=1, \ldots, t . p_{l t}$ denotes manager $l$ 's posterior at 
time $t$ if prior to manager t's effort decision $T_{t}=\{o\}$ and if manager $l$ at his time of entry did not exert effort. Consider manager $l$ at time $t$. What induces manager $l$ to exert effort at time $t$ ? Is $p_{l t} \gamma-e \leq\left(1-Z_{t}\right) p_{l t} \gamma$ ? Suppose first that $l=t-1$. Note that $p_{t-1 t-1}=p_{t-1 t}$ (manager $t-1$ knows that he did not exert any effort at time $t-1$, therefore his act of adopting $o$ doesn't affect his posterior probability concerning the existence of $n)$. Now $p_{t-1 t} \gamma-e<p_{t-1 t-1}-e=\left(1-Z_{t}\right) p_{t-1 t} \gamma$. Hence, due to switching costs manager $t-1$ strictly prefers not to exert effort at time $t$. Assume next that $l<t-1$. Manager $l$ doesn't want to exert effort at time $t$ either if (s.c.) $p_{l t} \gamma-e \leq p_{t-1 t} \gamma-e$ or if $p_{l t} \leq p_{t-1 t}$. Now,

$p_{l t}=\frac{z_{1} z_{2} \ldots z_{l-1} z_{l+1} \ldots z_{t-1}}{z_{1} z_{2} \ldots z_{l-1} z_{l+1} \ldots z_{t-1} p+(1-p)} p \leq \frac{z_{1} z_{2} \ldots z_{l-1} z_{l} z_{l+1} \ldots z_{t-2}}{z_{1} z_{2} \ldots z_{l-1} z_{l} z_{l+1} \ldots z_{t-2} p+(1-p)} p=p_{t-1 t}$

if and only if $z_{t-1} \leq z_{l}$ or iff $q_{t-1} \geq q_{l}$. From proposition (4) we know that this is the case as soon as $\gamma \leq 1-e$.

Consider now all managers at time $K+1$ and assume that everyone adopted $o$. Using a reasonning similar to our previous one, the reader can easily check that if $\gamma \leq 1-e$, manager $t(t<N)$ doesn't want to exert effort at time $K+1$ because $p_{t K+1} \leq p_{N}=e$. Q.E.D. 